\title{
Evaluating Navigational Surrogate Formats with Divergent Browsing Tasks
}

\author{
Andruid Kerne ${ }^{1}$, Steven M. Smith ${ }^{2}$ Hyun Choi $^{2}$, Ross Graeber ${ }^{1}$, Daniel Caruso ${ }^{1}$ \\ Department of Computer Science ${ }^{1}+$ Department of Psychology ${ }^{2}$ [Interface Ecology Lab] \\ Texas A\&M University \\ College Station, TX 77843, USA \\ andruid@cs.tamu.edu, \{stevesmith, hyun-choi, rgraeber\}@tamu.edu, dcaruso@csdl.tamu.edu
}

\begin{abstract}
Navigational surrogates are representations that stand for information resources within search engine result sets, e-commerce sites, and digital libraries. They also form the basis of personal collections of media, such as web pages. Our hypothesis is that the formats of individual surrogates and collections play an important role in how people use collections. We are particularly interested in processes of information discovery, in which ideas are iteratively reformulated in the context of working with information.

To investigate how the representation of navigational surrogates affects how people work with information, we have created a collection of undergraduate psychology curriculum resources in 3 alternative formats: a linear list of textual elements, a spatialized set of textual elements, and a spatialized set of labeled images that have been composited. To evaluate navigation with these surrogate formats during information discovery, we designed divergent browsing tasks, that is, tasks that require assembling information from multiple diverse sources. A within-subjects evaluation indicates that users prefer the spatial labeled images format, and navigate more effectively with it.
\end{abstract}

\section{Author Keywords}

surrogates, navigation, collections, divergent thinking, evaluation, information discovery, creative ideation

\section{ACM Classification Keywords}

H.5.2 Information Interfaces and Presentation (e.g., HCI): User Interface; Evaluation/methodology

\section{INTRODUCTION}

As people spend more time working with digital libraries, engaging in e-commerce, and searching the web, the importance of the format of the navigational elements that they encounter grows. According to Morrison, Pirolli and Card's analysis of the Georgia Tech Graphics, Visualization, and Usability Center web usage survey [5], the reason why people use the web in $69 \%$ of cases is to understand or

Copyright is held by the author/owner(s).

CHI 2005, April 2-7, 2005, Portland, Oregon, USA.

ACM 1-59593-002-7/05/0004. compare/choose [11]. The method of users in $71 \%$ of cases, is to collect [ibid], that is, to assemble information from multiple sources. Scenarios such as comparison shopping and citation collecting are prevalent and important. In these scenarios, users need to consider the connections among many diverse information resources. Of particular interest are processes of information discovery, in which new ideas are formed and iteratively reformulated in the context of the stimulus of information. [9]. We are beginning to investigate how users work with different navigational element, i.e., surrogate, formats, to understand how best to build tools to support navigation for information discovery. Such tools include environments for collecting and browsing, as well as result-set generators.

In conducting this investigation, using a controlled study, we want to define tasks that match the cognitive processes found in real world scenarios. Prior studies in most cases have focused on convergent thinking tasks. Convergent thinking tasks use well-defined problems with unique correct answers. Divergent thinking tasks are less well-defined, and demand a variety of creative responses that might vary in terms of practicality [4]. By divergent browsing tasks, we mean divergent thinking tasks which require browsing to assemble information resources from multiple diverse sources in order to form answers. Although convergent tasks are easier to construct for experiments, and their solutions are easier to score, divergent browsing tasks, in which users explore multiple diverse information resources seeking useful discoveries, may better exemplify common and important web usage scenarios.

\section{NAVIGATIONAL SURROGATES}

Surrogate is a term from library science. A surrogate is "a replacement for an original item, ... which gives some description of the item, and how it can be obtained" [1]. Ding and Marchionini applied the term to digital libraries while constructing surrogates for video [3]. Examples of digital surrogates include Amazon catalogue entries, ACM digital library entries, search engine result set elements (e.g., Google gists), and bookmarks. 


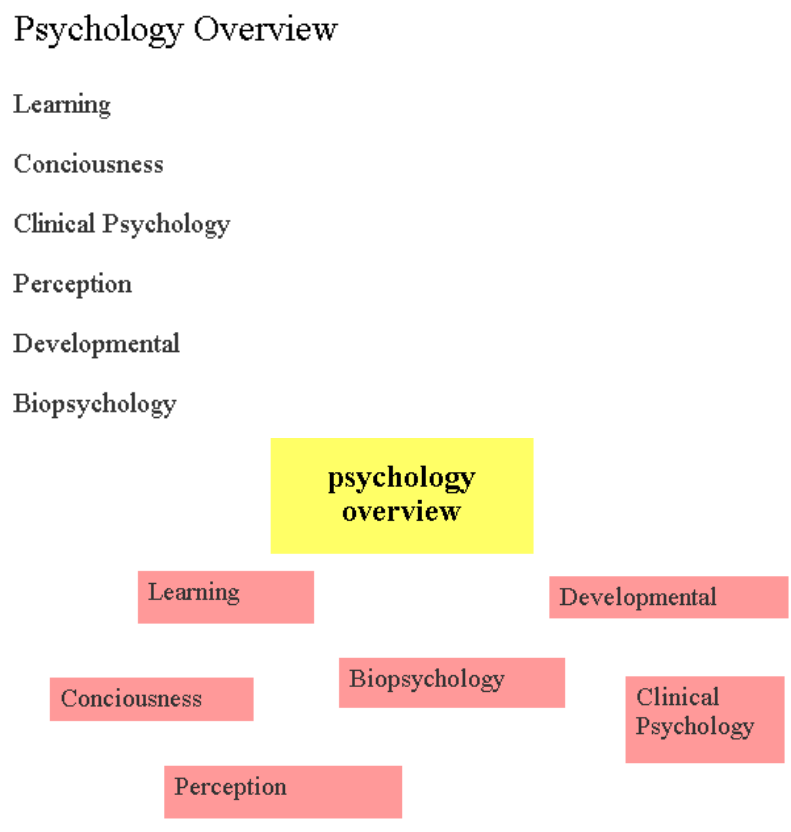

Figure 1: Linear text and spatial text formats of the Psychology Overview Collection

We are working with three formats of surrogate collections. The first is the linear list of text elements (figure 1). This form is the one most used in prevailing practice. It is typified by the bookmarks menu. The second format is a spatialized collection of textual elements (figure 1). This is the form of spatial hypertext [10]. Spatial arrangement can help users see relationships [ibid].

The third format is spatial labeled images (figure 2). Thumbnail images have previously been used to represent surrogates [e.g., 7, 12]. Woodruff et al advanced this approach by creating "enhanced thumbnails," in which the words associated with a search term are enlarged [15]. Our labeled images are similar to enhanced thumbnails, except that we forego the thumbnail overview of a document's appearance; instead, we extract a representative image from the document and superimpose a representative text. The text is rendered with a form of stroking, which creates hue and value contrast [6] to ensure legibility, while minimizing how much of the image is obscured.

\section{EXPERIMENTAL APPROACH}

In the present study, rather than making omnibus comparisons of navigational systems, we used an experimental approach to ask questions about navigational surrogate representations, examining the efficacy of specific dimensions of the surrogates. Two dimensions, the representational format of each surrogate (text vs. labeled image), and the spatial layout of the collection (serial lists vs. spatially arranged clusters), were singled out and examined. Both dimensions were hypothesized to be relevant to the user's cognitive abilities, such that spatial clusters would augment the formation of "chunks" in working memory [13], and labeled images would augment the rapid formation of mental models of the information resources linked to the

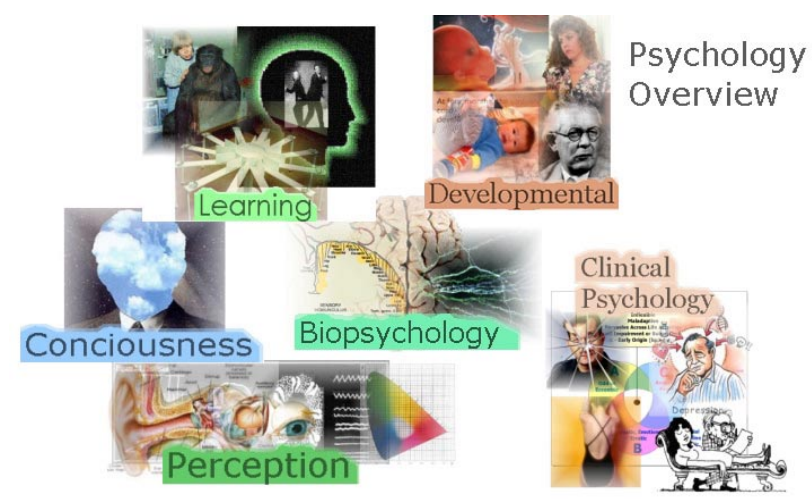

Figure 2: Composited labeled images format of the Psychology Overview Collection.

surrogates [9].

Some prior studies have used search tasks over wide, unclassified sets of web pages [e.g., 15]. To isolate navigational surrogate format from the effects of dynamic components, such as search engines, the present study focuses on browsing, rather than searching. We also wanted to include authored collections in the scope of inquiry. To integrate these factors, we authored a specific collection for this study, and developed equivalent versions in each of the 3 navigational surrogate formats. We designed divergent browsing tasks specifically to work with this collection.

\section{A Psychology Resources Collection}

We used our university's psychology pool as a source of subjects for the experiment. These subjects are undergraduates in the introductory psychology class, who fulfill a course requirement for learning about what a psychology study is by participating in one as a subject. To maximize the educational value of our collection-authoring efforts, we assembled information resources based on the psychology curriculum. We intend to make this collection available to the community, as a pedagogical resource.

We divided the psychology curriculum into 12 topics. We collected information resources for 6 of these topics: biopsychology, learning, developmental, consciousness, clinical psychology, and perception. The resources were gathered from diverse sources, including the websites of governmental agencies, medical centers, research institutes, internet journals, and university departments.

We created equivalent surrogates and authored equivalent collections of psychology information resources, using each of the 3 surrogate formats: linear text, spatial text, and spatial labeled images. First, the set of information resources was gathered. Then, we used combinFormation [8] to author the collections. We started with the most complex format, spatial labeled images. A representative image was selected from each resource website. The image was dragged from Firefox into a combinFormation workspace. An appropriate text label was either extracted from the source document or created. Together, the image and text label comprise a surrogate. Surrogates were positioned within the information space based on mutual relationships. Sizes of elements and colors 
for text stroking were chosen to maximize layering and legibility $[14,6]$. In some cases, images were processed with translucent border areas in order to visually represent strong interconnections between groups of information resources. While combinFormation saves information spaces primarily as XML, we utilized the program's auxiliary images+text HTML output format to derive a simplest product to use in the study. We added to combinFormation a mechanism for automatically deriving spatialized text and linear text collection formats from an images+text collection, and outputting these as HTML.

In the authored collections, navigation is accomplished by clicking on a surrogate. Each collection begins with a Psychology Overview, consisting of surrogates for each of the 6 topics (figures 1-2). Navigation from here leads to the topic-specific collections, each of which contains an additional 6-10 surrogates. Clicking within each topic collection leads to the information resources, themselves. For example, within the "Learning" collection, clicking on the surrogate for "Neuroplasticity" leads to a site developed as part of NIH's Science Education Partnership [2].

\section{Experimental Method}

There were three divergent browsing questions (e.g., "What kinds of things can cause behavioral problems for children in school?"), which were manipulated within-subjects. Each question was designed to be answered by navigating the collection of psychology resources. The instructions for each question stated, "Gather all of the possible facts relevant to this topic from the websites provided, and list the facts. For each fact write a brief description (e.g., one sentence) of its relevance to the topic. List as many varied and unusual facts as you can."

Each subject received one divergent question in each format. The order of the 3 questions and the 3 formats of navigational surrogates was counterbalanced between subjects, thereby producing 9 different combinations of question order and surrogate formats. Each participant was assigned to one of these counterbalancing conditions.

Fifty-two introductory psychology students were recruited using an internet signup system. Each session was held in a group of 5-15 participants at a time. Participants were asked to find answers for questions chosen from various areas of psychology, by browsing websites that include possible answers. They were told to give as many answers as they could find using the time provided. They were informed that answers for the questions either could be found in a single website, or gathered from several websites. The experiment consisted of three parts: pre-experimental questions, the three divergent questions, and post-experimental questions. Participants answered two brief pre-experimental questions as to the frequency of their internet use and their gender before they started answering the divergent questions. Pressing a start button led to a framed webpage in which each question was presented in the top frame. There was also an "Overview" button in the upper right hand corner, for browsing the Psychology Overview (figures 1,2) in the bottom frame. Each divergent question was followed by two questions about the experience of answering it (e.g., "How challenging was answering the last question?"). Participants were given 8 minutes for each divergent question. They could see the time remaining counting down on a digital clock. At the end of eight minutes, if the participants had not clicked "submit," the program automatically submitted what they had written, and the next question was displayed. After all three divergent questions were answered, participants answered post-experimental questions about their preference among the three navigational formats, and the reasons for their choices. The whole procedure lasted approximately 40 minutes.

To avoid the variability that would be caused by Internet download times, resource websites were mirrored locally on our server. Mirroring these sites also facilitated construction of a servlet that mediated requests for the resources. This servlet acted as a routing system, and as a means for logging participants' navigation paths.

\section{RESULTS}

A 3 (surrogate format) X 2 (navigation level) ANOVA was computed to examine the frequency of browsing pages at the authored collection level vs. the actual information resource websites linked to by these collections. Although the main effect of surrogate format was not significant $[F(2,51)<1.0]$, the effect of navigation level was significant $[F(2,51)=$ $14.08, p<.01$ ]; more information resource websites than topic-based collections were browsed (figure 3). More importantly, the surrogate format $\mathrm{X}$ navigation level interaction was significant $[F(2,102)=3.71, p<.05]$; pairwise $t$-tests showed that the interaction was due to the fact that the number of information resource websites browsed significantly exceeded collection level visits only for the spatial labeled image surrogate format. This result indicates that the use of information resources linked to by the topic-based collections was enhanced through the use of spatially arranged labeled images.

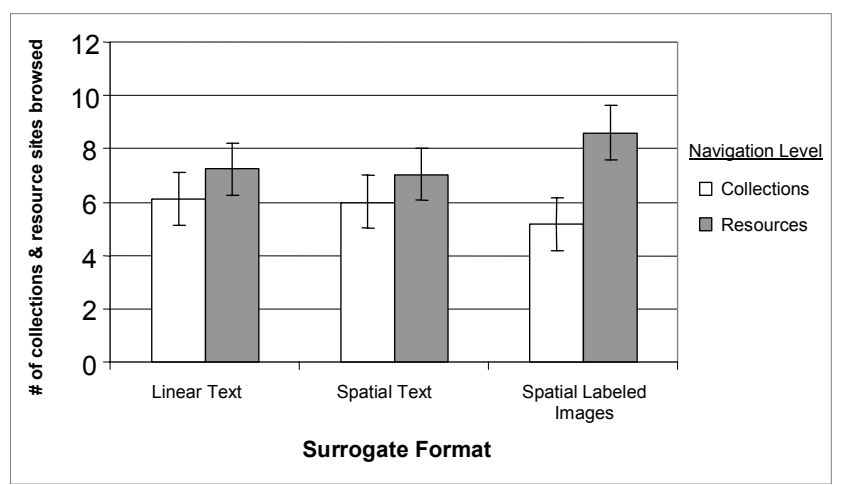

Figure 3: Browsing of the authored collections vs. information resources as a function of surrogate format. 


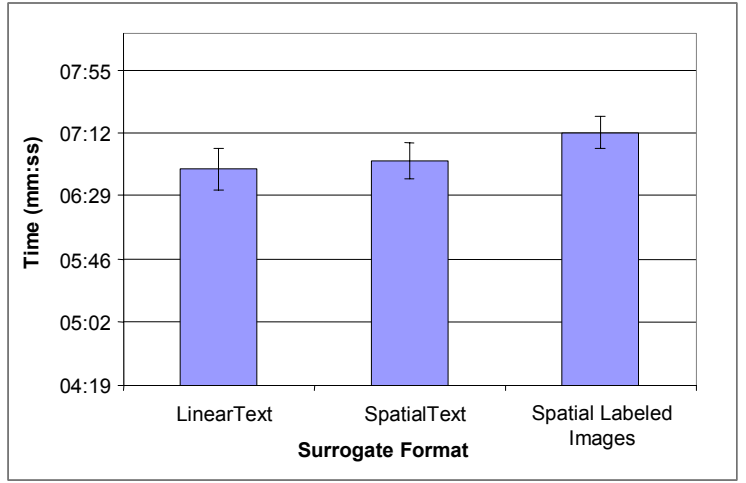

Figure 4: Time spent developing answers to each question (persistence) as a function of surrogate format.

Consistent with the navigation level browsing data were the results regarding persistence in developing answers (fig. 4). Participants spent more time on the divergent tasks when they used the spatial image format, as compared to the other two text formats. This indicates greater attention. Although there was a trend indicating that participants chose to spend more time on a task when they used spatial labeled images, the effect did not reach significance $[F(1,52)<1.0]$.

In addition to the behavioral data, we collected subjective measures of user experience in relation to the three surrogate formats, liking, ease of use, and utility (figure 5). Participants clearly liked the spatial labeled image surrogates best $\left[X^{2}(2)\right.$ $=34.88, p<.001]$; over $70 \%$ preferred that format. Linear texts were preferred second most, and spatial text surrogates were the least preferred. Further, participants found the image surrogate format easiest to use of the three $\left[X^{2}(2)=\right.$ $16.12, p<.001]$, with linear texts second and spatial texts most difficult. Spatial image surrogates were also seen as most useful, with linear texts second and spatial text surrogates least useful $\left[X^{2}(2)=29.58, p<.001\right]$. Thus, all three experience measures show that participants preferred the spatial image format.

\section{CONCLUSIONS}

The behavioral results and subjective experience data tell the same story. Users engaged in divergent browsing tasks preferred spatial labeled images, and found this navigational surrogate format to be easiest to use and most helpful. Further, when they used spatial labeled images, users spent more time developing answers, and browsed more information resources. They visited the collection level less. We interpret this to mean that the spatial labeled images format of navigational surrogates facilitated the formation of mental models of the information resources. Users were able to spend less time considering the collection level, and more time with the information resources themselves. This constitutes more effective navigation and use of cognitive resources [9]. We conclude that navigation and information discovery will be improved by composition, generation, and browsing tools that represent collections in the spatial labeled images format. The more familiar format, linear text, was a distant second-best. Spatial text was least preferred and least useful.

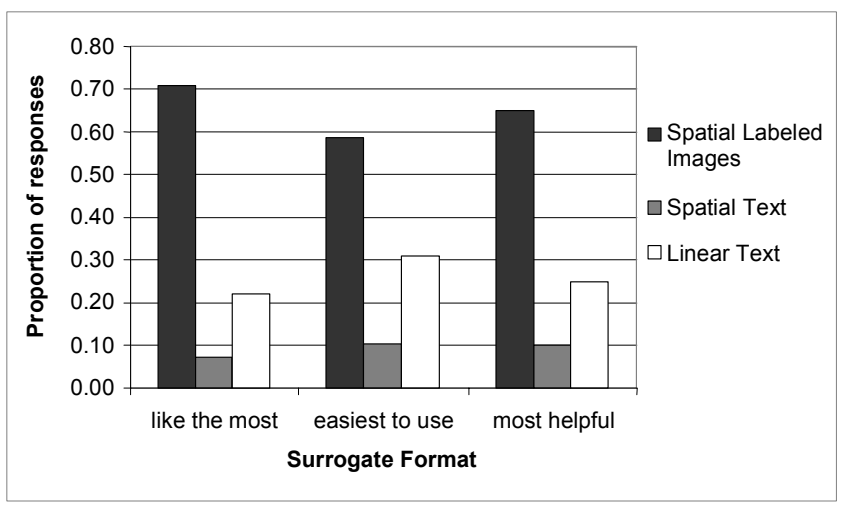

Figure 5: Subjective measures: liking (preference), ease of use, and helpfulness as a function of surrogate format

\section{ACKNOWLEDGMENTS}

Support has been provided by NSF grant IIS-0429469.

\section{REFERENCES}

1. Burke, M., Organization of Multimedia Resources, Hampshire, UK: Gower, 1999.

2. Chudler, E., Neuroscience for Kids, 2004, http://faculty.washington.edu/chudler/neurok.html.

3. Ding, W., Marchionini, G., Soergel, D., Multimodal Surrogates for Video Browsing, Proc DL 1999, 85-93.

4. Finke, R., Ward, T., Smith, S. Creative Cognition. Cambridge MA., MIT Press, 1992.

5. Georgia Tech GVU, GVU's lOth WWW User Survey, http://www.gvu.gatech.edu/gvu/user_surveys/, 1998.

6. Itten, J. The Elements of Color, New York: VNR, 1962.

7. Kaasten, S., Greenberg, S., Integrating Back, History and Bookmarks in Web Browsers, Proc CHI 2001 Extended, 379-380.

8. Kerne, A., Mistrot, J.M., Khandelwal, M., Sundaram, V., Koh, E., Using Composition to Re-Present Personal Collections of Hypersigns, Proc CoSIGN 2004.

9. Kerne, A., Smith, S., The Information Discovery Framework, Proc DIS 2004, 357-360.

10. Marshall, C.C., Shipman, F.M., VIKI: Spatial hypertext supporting emergent structure, Proc ECHT 1994, 13-23.

11. Morrison, J.B., Pirolli, P., Card, S.K., A Taxonomic Analysis of What World Wide Web Activities Significantly Impact People's Decisions and Actions, Proc CHI 2001 Extended, 163-164.

12. Robertson, G., Czerwinski, M., Larson, K., Robbins, D.C., et al, Data Mountain, Proc UIST 1992, 153-162.

13. Simon, H.A., How big is a chunk?, Science, 183, 1975.

14. Tufte, E., Envisioning Information, Cheshire, CT: Graphics Press, 1990.

15. Woodruff, A. Rosenholtz R., Morrison, J., Faulring, A., Pirolli, P., A Comparison of the Use of Text Summaries, Plain Thumbnails, and Enhanced Thumbnails for Web Search Tasks, JASIST 53(2):172-185, 2002. 